Int. J. Dev. Biol. 50: 39-46 (2006)

doi: $10.1387 / \mathrm{ijdb} .052068 \mathrm{zz}$

Original Article

\title{
The expression and alternative splicing of alpha-neurexins during Xenopus development
}

\author{
ZHIHONG ZENG ${ }^{1, \#, ~ C O L I N ~ R . ~ S H A R P E ~}{ }^{2}$, J. PAUL SIMONS ${ }^{3}$ and DARIUSZ C. GÓRECKI*,1 \\ ${ }^{1}$ School of Pharmacy and Biomedical Sciences, ${ }^{2}$ School of Biological Sciences, Institute of Biomedical and Biomolecular Sciences, \\ University of Portsmouth, U.K. and ${ }^{3}$ Department of Anatomy and Developmental Biology, University College London, \\ Hampstead Campus, London, U.K.
}

\begin{abstract}
The neurexins are involved in the formation and function of synapses. Each of three genes encodes $\alpha$ - and $\beta$-neurexins. Additional diversity (particularly of $\alpha$-neurexins) arises from alternative splicing, resulting in a large number of protein isoforms, the significance of which is currently unclear. We have analysed $\alpha$ neurexin expression and alternative splicing during development of the frog Xenopus laevis. Surprisingly, each $\alpha$-neurexin gene is expressed in immature oocytes. During embryonic development, each Xenopus neurexin (nrxn) gene has a distinct temporal expression pattern, with expression being almost exclusively within the neuroepithelium. The spatial expression of $n r x n l \alpha$ and $n r x n l l \alpha$ is similar in the developing CNS, with staining being observed in the optic cup and in dorsolateral regions of anterior neural tube, but not adjacent to the ventral midline. The pattern of nrxnIII $\alpha$ expression is more restricted, in several domains of the anterior neural tube. In the forebrain, expression was confined to an area in the ventrolateral neural tube; nrxnIIl $\alpha$ was also expressed in the hindbrain and spinal cord. By stage 32, a period when synaptogenesis occurs, nrxnIIl $\alpha$ is expressed midway along the neural tube's dorso-ventral axis in the hindbrain and anterior spinal cord, at the site of the primary interneuron column. Because of the striking diversity of neurexin isoforms, we analysed alternative splicing of Xenopus transcripts during development and found examples of alternative splice variants of each neurexin. The data demonstrate differential regulation of the $\alpha$ neurexins with respect to the gene temporal and spatial expression and alternative splicing.
\end{abstract}

KEY WORDS: alternative splicing, Xenopus, development, neurexin, primary neuron

\section{Introduction}

The formation of a functional neural circuit requires directed axon growth (pathfinding) and the formation of specialised contacts with appropriate target cells (synaptogenesis). In many cases, more neurons than required will initiate these processes, so regulation of cell number is also important (reviewed in Albright et al, 2000). While a great deal is now known about the molecular mechanisms of pathfinding and control of cell number, less is known about synaptogenesis although it is clearly an important event during CNS development. In addition, the observed plasticity of neuronal connections in the CNS indicates that mechanisms for synaptogenesis are not restricted to development but are retained by the mature nervous system (Zito and Svoboda 2002).

The formation of a synapse involves specific interactions between proteins at the neuronal cell surface and the environ- ment, whether this is the surface of another cell, the extracellular matrix or a diffusible signalling protein. The question remains, how can these proteins generate the diversity needed to recognise just one from the very large number of potential target cell types? Recent analyses suggest that diversity in the CNS can be generated, at least in part, through alternative splicing that generates large numbers of related, but distinct, transcripts and hence proteins from a single gene (Schmucker et al, 2000). That these proteins could have individual functions has been demonstrated for the different isoforms of Dscam, which each possess specific binding affinities (Schmucker et al, 2000). Mammalian neurexin (nrxn) genes (Ushkaryov et al, 1992, 1994, Ullrich et al, 1995) potentially encode over a thousand different isoforms of a cell

Abbreviations used in this paper: EGF, epidermal growth factor; nrxn, neurexin; $\mathrm{SP}$, signal peptide.

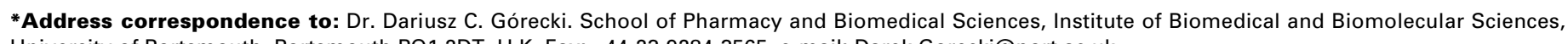
University of Portsmouth, Portsmouth P01 2DT, U.K. Fax: +44-23-9284-3565. e-mail: Darek.Gorecki@port.ac.uk

\# Present address: Genome Damage and Stability Centre, University of Sussex, Brighton, BN1 9RQ, U.K. 
surface protein, found predominantly on neurons. Neurexins are encoded by three genes (I, II and III) and each gene is controlled by two promoters (Ushkaryov et al, 1992, 1994) resulting in the major $\alpha$ and $\beta$ isoforms, with additional diversity generated by alternative splicing of the primary transcripts (Rowen et al., 2002; Tabuchi and Sudhof, 2002).

Neurexin immunoreactivity has been localised to pre-synaptic nerve terminals (Dean et al, 2003) while known ligands (including dystroglycan and the neuroligins) are found predominantly at post-synaptic sites in target cells (Górecki et al, 1994; Song et al., 1999; Sugita et al., 2001; Zaccaria et al., 2001). Significantly, contact with neuroligin-expressing non-neurona/cells is sufficient to induce the formation of pre-synaptic terminals in neurones via neurexin-mediated mechanisms (Nguyen and Südhof 1997; Scheiffele et al., 2000; Dean et al., 2003). Indeed, recent findings indicate that the $\beta$-neurexin-neuroligin link is a core component mediating the formation of presynaptic and postsynaptic specialisations of both GABAergic and glutamatergic synaptogenesis (Graf et al, 2004).

While there is alternative splicing of $\beta$-neurexins, by far the greatest number of splice variants exist for $\alpha$-neurexins; the $\alpha$ neurexin amino acid sequences, including those of the variant

A

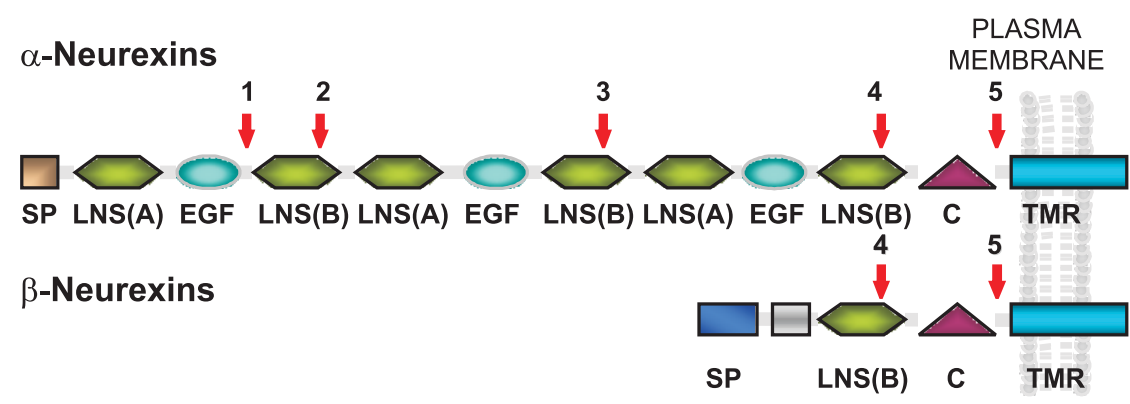

B

nrxn 2

$\operatorname{nrxn} 3$

nrxn 1

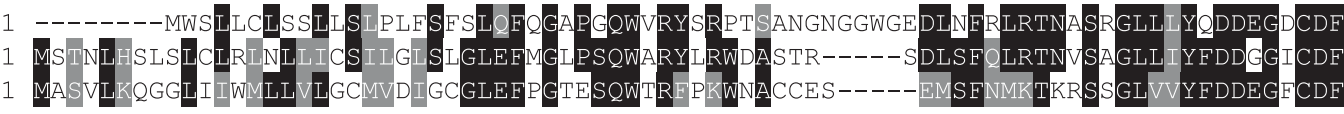

nrxn 2

nrxn 3

nrxn 1
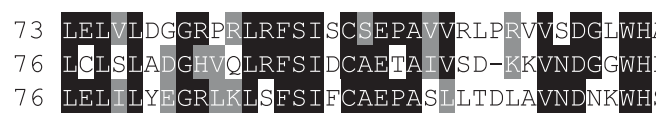

III

鄗

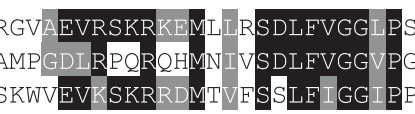

nrxn 2

nrxn 3

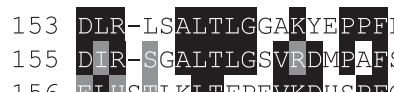

nrxn 1

156

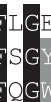

nrxn 2

nrxn 3

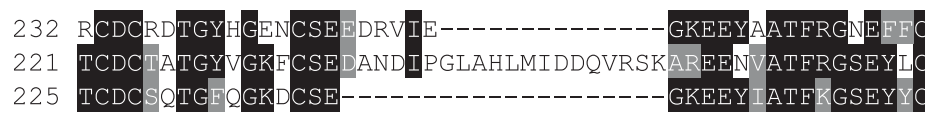

YDLSHSPIQSSADEITLSFRTLQRNGLM

nrxn 1

221

225

GKEEYIATEKGSEY

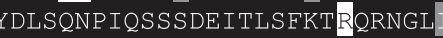

$\operatorname{nrxn} 2$

298 LHTGKSADYVNLSLK

301 LHTGKSADYVNLAL

285 LHTGKSADYVNLALKN
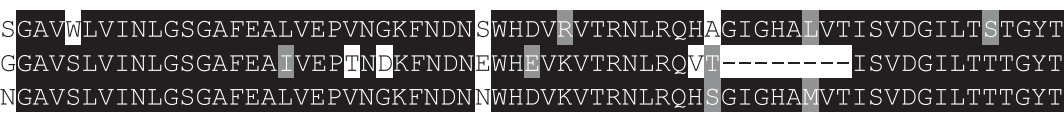

\section{C}

nrxn 11410 AMYKYRNRDEGSYHVDESRNYISNSAQSNGAVIKEKQPSSAKSSNKNKKNKDKEYY 1465

nrXn 21630 AMYKYRNRDEGSYQVDQSRNYISNSAQSNGAVVKEKTTPATKMAGKSKKNKDKEYY 1685

nrXn 31410 AMYKYRNRDEGSYQVDESRNYI TNSAQSNGA VKKEKQ-HSSKGNHSKQKNKDKEYY 1464

Fig. 1. Representation of the domain structure and alternative splicing sites of neurexins. Modified from Missler and Sudhof (1998). (A) Diagram shows proteins schematically, with the extracellular portion on the left-hand side. Abbreviations: SP, signal peptide; LNS(A), LNS(B), two types of laminin/neurexin/sex hormone-binding globulin domain; EGF, epidermal growth factor-like domain; C, carbohydrate attachment site; TMR, transmembrane domain. Arrows indicate positions of variable regions generated by the alternative splicing of transcripts. (B,C) Protein sequence alignments of Xenopus $\alpha$-neurexins I, II and III. (B) Sequences of the N-terminal regions. Note the inserts generated by alternative splicing in nrxn III, splice site 1 and nrxn I and II, site 2. (C) Alignment of the highly conserved transmembrane and cytoplasmic regions. 
TABLE 1

COMPARISON OF THE PREDICTED PROTEIN SEQUENCES

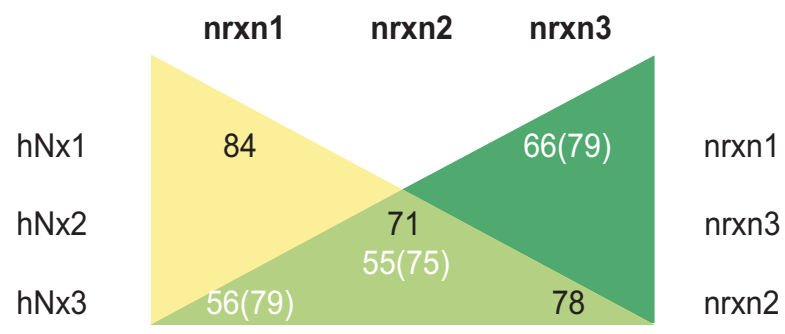

Comparison of the predicted protein sequences (expressed as \% of homology) between each Xenopus neurexin ( $\mathrm{Nrxn}$ ) and its respective human orthologue ( $\mathrm{hNx}$, in bold). In brackets: homology between the most highly conserved transmembrane regions of Xenopus neurexins. Also see: Fig. 1.

isoforms, are highly conserved between orthologues in mammals, birds and fish and some of the alternatively spliced exons are also conserved between paralogous genes (Rowen et al, 2002; Tabuchi and Sudhof, 2002). In addition, changes in neurexinIl $\alpha$ expression during synaptogenesis, in synaptic remodelling in the mouse brain (Górecki et al, 1999) and following ischaemia (Sun et al., 2000) support a role for $\alpha$-neurexins during synaptogenesis. However, the precise functions of the $\alpha$-neurexins and the significance of the many isoforms generated by alternative splicing remain to be determined.

Amphibian larvae co-ordinate their initial movements with a subset of neurons called the embryonic primary neurones. When first active, there are around 250 embryonic primary sensory neurones (Sharpe and Goldstone 2000 a,b) and probably fewer inter and motorneurones, each localised to specific, easily recognized domains. The simple arrangement of embryonic primary neurones within the neural tube, combined with the accessibility of Xenopusembryos, makes this an excellent system to examine the earliest steps in neurone formation (Sharpe and Goldstone 1997; Goldstone and Sharpe 1998) whilst providing an insight into more complex mammalian nervous systems. For this reason we have chosen to characterise Xenopus neurexins and their expression in embryos.

In this paper we show that the $\alpha$-neurexins are expressed in the oocyte and that expression of each gene shows a distinct specific spatio-temporal pattern with alternatively spliced isoforms that are present only in the late embryo, coincident with the formation of a functional nervous system.

\section{Results}

\section{Molecular characteristics of Xenopus laevis neurexins}

Initially we examined the range of neurexins expressed in $X$. laevis. To obtain partial sequences of $X$. laevis neurexin cDNAs, degenerate primers complementary to the conserved cytoplasmic domains of neurexins I, II and III were used for RT-PCR amplification of adult $X$. laevis brain transcripts. The PCR products, cloned and sequenced were identified as neurexins I, II and III.

Comparison of rat neurexin sequences with the $X$. tropicalis genome revealed just three independent scaffolds (\#170 [66\% identity], \#111[ 48\%] and \#145 [ 40\%]) indicating that there are three neurexin genes in frogs. The neurexin PCR products were then used to screen a $X$. laevis cDNA library and a full-length neurexin II $\alpha$ clone and partial neurexin I $\alpha$ and III $\alpha$ clones were isolated and sequenced. This information and sequences obtained from EST databases aided the design of specific primers that, combined with PCR amplification, allowed cloning of the full coding sequences of $X$. laevis neurexins I and III $\alpha$ (database submission in progress).

The predicted protein sequence homology between frog and mammalian neurexins was high (71-84\% identity) and as expected, the similarity between neurexin orthologues from frog and mammal (human) was much higher than the degree of similarity between paralogous Xenopus neurexins (Table 1). This confirms the highly conserved nature of these proteins in different vertebrate species and the high sequence specificity for each neurexin within the family.

\section{Each Xenopus nrxn gene has a distinct temporal pattern of expression}

Specific primers were used to amplify $\alpha$-neurexin transcripts from each gene (Fig. 2). Although similar, each gene had its own distinct temporal pattern of expression. Surprisingly, transcripts from each neurexin gene were found in the oocyte. Although the maternal nrxnl transcripts were not seen following fertilization (stage 1, see Fig. 2), nrxnll and nrxnlll transcripts persisted into the early embryo but with decreased abundance during the blastula and gastrula stages. The level of nrxnl and nrxnll transcripts increased in the early tailbud (stage 24), which corresponds to the developmental stage at which functional, connected neurons differentiate (Jacobsen and Huang, 1985). Following the initial loss of maternal nrxnlll transcripts, their abun-
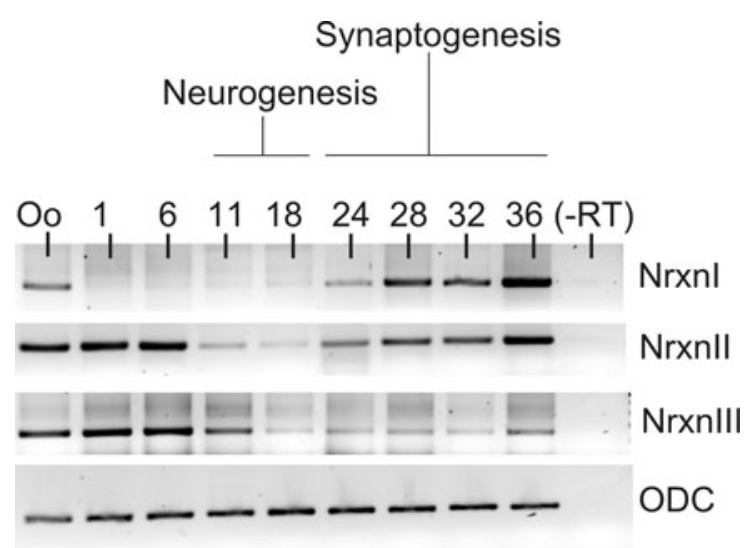

Fig. 2. Developmental series RT-PCR showing the temporal pattern of $\boldsymbol{\alpha}$-nrxn expression. Primers specific for each nrxn alpha cDNA were used to identify transcripts in Xenopus oocytes and across early development. Transcripts from each nrxn are found in the oocyte. However zygotic expression of $\mathrm{nrxn} I$ is first apparent in the early tailbud embryo (stage 24), which correlates with the onset of synaptogenesis. Transcripts for nrxn II and III are present in the early embryo, but whereas the level of nrxn II increases during tailbud stages, those of nrxn III show no marked increase by the tailbud stage. Consequently, each gene shows a specific temporal pattern of expression. ODC is used as a control for equivalent loading. Oo, oocytes; numbers correspond to stages of Xenopus development (Nieuwkoop and Faber, 1994) where 1 is the fertilised egg; 10 , the onset of gastrulation; 18, the late neurula stage; 28 , the tailbud stage and 36 is a swimming tadpole. 
dance remained relatively constant between stages 18 and 32 , but at a lower level than that of nrxns I and II.

\section{Neurexin expression in the oocyte}

RT-PCR analysis showed that transcripts corresponding to each neurexin gene are found in oocytes from which the follicle cells had been removed by collagenase. To demonstrate that expression was in the oocytes rather than in residual follicle cells, samples of ovary, containing oocytes at each stage of development, were subject to in situhybridisation with antisense and sense (control) neurexin probes (Fig. 3 ). In each case it is clear that nrxn transcripts are found within the oocyte cytoplasm and staining is most intense in immature oocytes. There is no evidence for nrxn expression in the follicle cells that surround the oocyte in the ovary. The decrease in signal at later stages probably represents a dilution of the mRNA in the

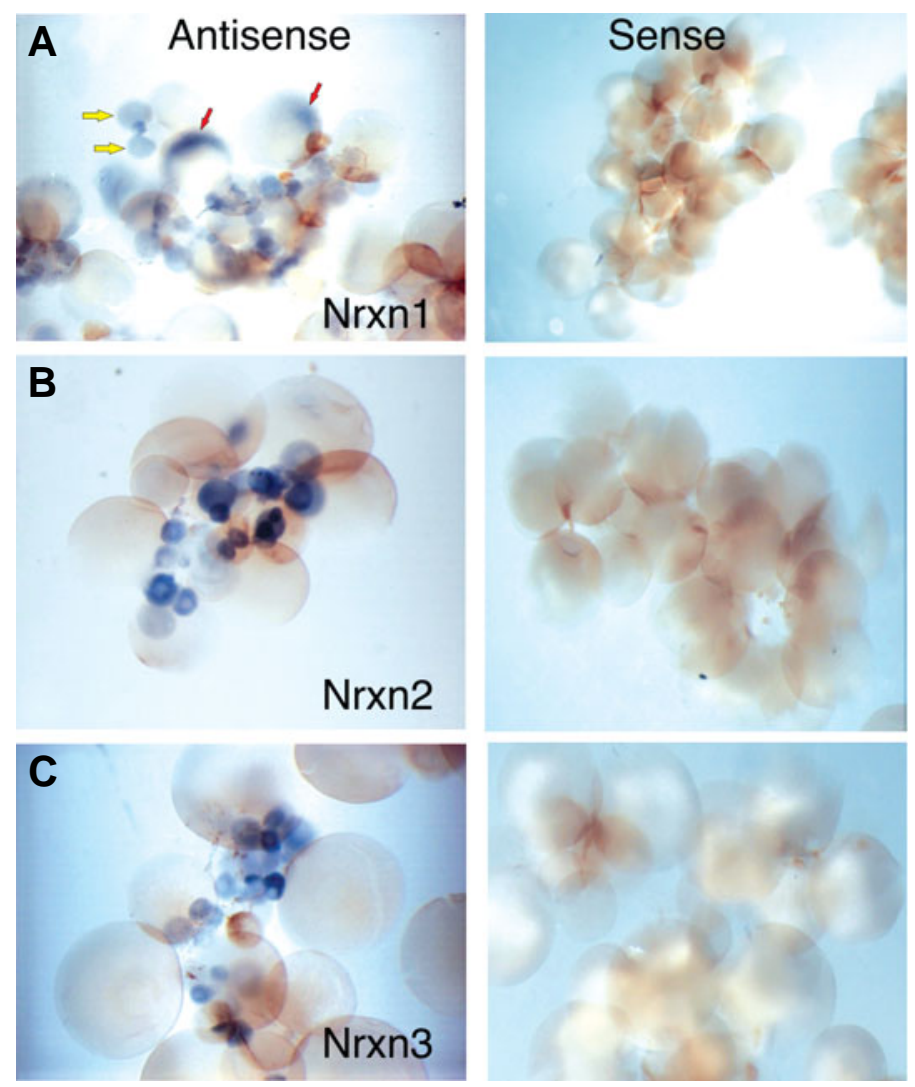

Fig. 3. Transcripts of $\alpha$-neurexins are expressed in Xenopus oocytes. Ovarian tissue consisting of connective tissue, follicle cells and oocytes at different stages of development was subject to wholemount in situ hybridisation using probes specific for each of the neurexin transcripts. (A) An nrxn I specific probe identifies transcripts equally distributed through small, early stage oocytes (yellow arrows). At later stages, the $\mathrm{nrxn}$ l $\alpha$ transcript is localised to a crescent within the large oocyte (red arrows). Transcripts were not detected in cells other than oocytes. (B,C) Nrxn II (B) and nrxn III (C) transcripts were found in all stages of oocyte. At the later stages, in the large oocyte, the signal is less intense probably due to the dilution of the mRNA in the larger cytoplasmic volume. There was no evidence for localisation of these transcripts and neither were they detected in cells other than oocytes. Right-hand-side panels: negative controls, hybridised with corresponding sense neurexin cRNA probes. expanded cytoplasmic compartment. Unlike nrxnll and nrxnllI, which are evenly distributed through the oocyte, $\mathrm{nrxnl}$ is localised to a crescent in the large, stage VI oocytes. Future experiments will determine whether this corresponds to a particular region of the oocyte, such as the animal pole or mitochondrial cloud.

\section{Spatial neurexin expression in the tailbud embryo}

Tailbud embryos that are capable of simple swimming movements coordinated by embryonic primary neuronal circuits (Roberts and Clarke, 1982) were analysed by in situhybridisation with gene-specific antisense neurexin alpha probes; the probes lacked the sequences held in common with the beta neurexins, so the signal detected shows specific expression of each alpha neurexin. Transcripts of each gene are detected almost exclusively within the neural tube. The spatial patterns of nrxnl $\alpha$ and nrxnll $\alpha$ were very similar and showed extensive staining in both the developing brain and spinal cord (Fig. 4). However, there was no staining along the midline or at the hindbrain-midbrain border. Transverse sections, taken at approximately the same antero-posterior levels, show that nrxnl $\alpha$ and nrxnll $\alpha$ expression is dorsolateral in the anterior neural tube but not adjacent to the ventral midline (Fig. 4 B, D). Both nrxnl $\alpha$ and nrxnll $\alpha$ are expressed in the optic cup, in prospective retinal cells.

In contrast, nrxnlll $\alpha$ has a more restricted pattern of expression. It is found in a limited number of domains in the anterior neural tube (Fig. 4E). Transverse sections show expression in the forebrain is restricted to a patch in the ventrolateral neural tube that does not extend as far dorsally as either nrxnl $\alpha$ or nrxnll $\alpha$ expression. Nrxnlll $\alpha$ was also expressed in the hindbrain and spinal cord. As nrxnlll $\alpha$ at these stages is expressed in subsets of neural cells, we focused our attention on this neurexin.

\section{Expression of neurexin IIl $\alpha$ is restricted to a subset of neuronal cells}

The first axons of embryonic primary neurones extend at stage 22, the first coordinated movements occur at stage 26 and the embryo can swim, when stimulated, by stage 32 . It is therefore likely that synaptogenesis of primary neurones will be taking place across these stages. In situ hybridisation with a nrxnlll $\alpha$ probe showed expression restricted to the hindbrain and anterior spinal cord at stage 24 (Fig 5A). A dorsal view showed staining in bilateral stripes that are likely to be primary interneurones and in small patches in the hindbrain (Fig 5B). We did not detect staining for nrxnlll $\alpha$ in the primary sensory or motor neurone columns at this stage. By stage 32, nrxnlll $\alpha$ expression was also seen in discrete patches within the anterior neural tube and hindbrain and in columns, which typically extends, in a posterior direction, approximately half way along the neural tube (Fig 5C). Close inspection indicates additional staining in a more ventral column of cells close to the midline though the position and dispersed nature of this pattern suggests these are not motor neurones. Sections of stage 32 embryos at the level of the hindbrain (Fig 5D) and the anterior spinal cord (Fig 5E) show localised nrxnlll $\alpha$ expression approximately midway along the dorsal ventral axis of the neural tube. This staining is consistent with nrxnlll $\alpha$ expression in the primary interneurone column. nrxnlll $\alpha$ staining is therefore seen in restricted regions of the neural tube consistent with its 


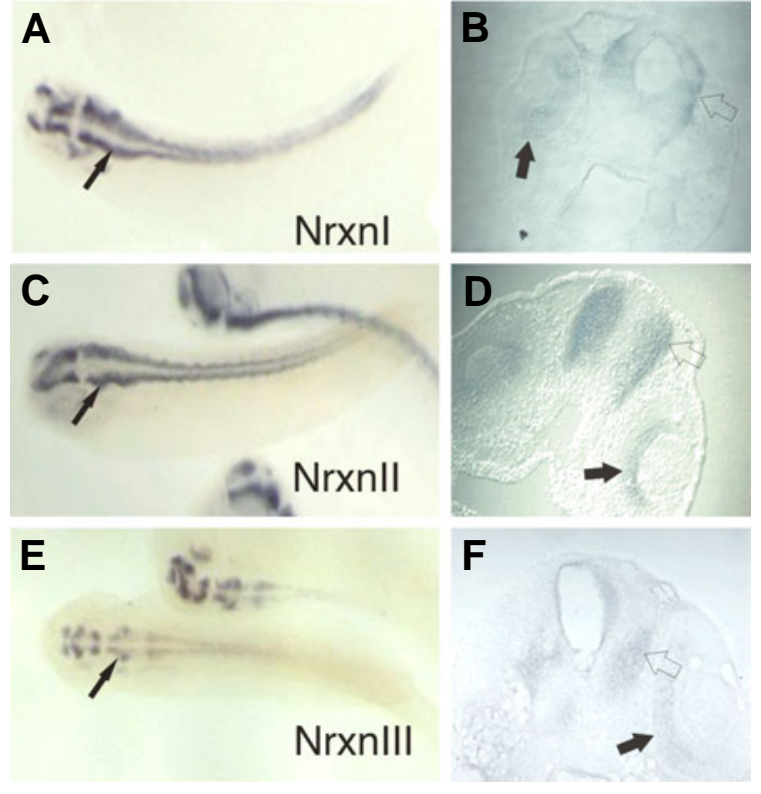

Fig.4. Expression of $\alpha$-neurexins is predominantly in neural tissue in the tailbud embryo. $(\mathbf{A}, \mathbf{B})$ In situ hybridisation with a nrxn l $\alpha$ probe showing whole embryo (A) and an anterior neural section (B). Nrxn I is found in cells along the length of the CNS but with a distinct gap at the midbrain-hindbrain boundary. Sections show expression in the dorsolateral neural tube and within the eye. (C,D) Embryos hybridised with a nrxn Il $\alpha$-specific probe show a similar pattern of staining to that seen with nrxn I. (E,F) Embryos hybridised with a nrxn IIl $\alpha$-specific probe show much more restricted expression in the CNS and in section show ventrolateral and eye expression in the anterior CNS (arrows).

expression in subsets of primary neurones at a time when synaptogenesis is taking place.

\section{Alternative splicing of neurexin transcripts during X. laevis embryonic development}

A key feature of neurexin expression in higher vertebrates is the production of numerous isoforms following the alternative splicing of primary transcripts. The identification of neurexin orthologues in Xenopus therefore prompted us to look for alternative splicing of the frog neurexins.

RNA was prepared from a developmental series and RTPCR was performed with primers designed to amplify across variant splice sites 1,2 and 3 , which are encoded upstream of the beta promoter and are therefore only represented in neurexin alpha isoforms. At each variant splice site in mammals there are a number of exons that can be included or excluded by alternative splicing. We have found examples of alternative splicing for each neurexin, but not at each variant splice site (Fig 6) and we have not detected alternative splicing at variant splice site 3 in any of the Xenopus neurexin genes at the stages of development examined. Because variant splice sites 4 and 5 are common both to alpha and beta neurexins, we would not be able to distinguish neurexin alpha and beta transcripts by RTPCR; for this reason we have not investigated splicing at these positions.

Transcripts from the nrxnl $\alpha$ gene showed alternative splicing at variant splice site 1 in the oocyte, but not in the embryo (Fig
$6 A)$. Sequence analysis revealed that the predominant transcript contained sequence that would encode a 10 amino acid sequence, GLAHLMMGDQ, which showed $100 \%$ homology to exon 4-encoded peptide in mouse neurexin I. The oocytespecific transcript lacked this sequence (Fig 6A). Alternative splicing in the nrxnll $\alpha$ gene was found at variant splice site 2 . The shorter form was predominant in early development while the longer form became prominent at later stages (stages 2436) (Fig 6B). The longer form encoded an insert of 7 amino acids (HAGIHAL) with extensive homology to the sequence encoded by exon $6 a$ of murine neurexin II $\alpha$ (HAGIGHAM). Finally, there was a complex pattern of alternative splicing at variant splice site 1 of nrxnlll $\alpha$. The predominant form, particularly abundant in oocytes and in the early embryonic stages lacked sequence corresponding to murine exons 2 and 3 . Two longer variants were characterised, the first included exon 2 and the second included both exons 2 and 3 (Fig. 6C).

\section{Discussion}

The neurexins are a highly conserved family of neuronal proteins encoded by three genes (I, II and III), each gene being controlled by two promoters to give the major $\alpha$ and $\beta$ isoforms. Based on previous results, which demonstrated changes in expression of $\alpha$-neurexins in mammalian synaptogenesis and synaptic remodelling (Górecki et al, 1999) and because the majority of the different neurexin variants are $\alpha$-neurexins, we focus our attention on the $\alpha$-isoforms. The inherent complexity of the vertebrate brain and the combinatorial expression of neurexin isoforms complicates studies on the functions of specific neurexins. Consequently, we have examined neurexin expression in the simple vertebrate nervous system of Xenopus laevis embryos. The nervous system of an amphibian embryo consists of a very small number of neurons called the embryonic primary neurones. When first active, there are around 250 embryonic primary sensory neurones (Sharpe and Goldstone $2000 \mathrm{a}, \mathrm{b}$ ) and probably fewer inter and motorneurones, each localised to specific domains. Using this model we have observed specific spatio-temporal expression patterns of $\alpha$ neurexins. For nrxnl $\alpha$ and nrxnll $\alpha$ the mRNA levels increase as the nervous systems begins to differentiate and produce functional, connected neurons. The spatial patterns of nrxnl $\alpha$ and nrxnll $\alpha$ expression were also similar with extensive dorsolateral domains in the anterior neural tube and in retinal cells. The expression of nrxnlll $\alpha$ was restricted to a subset of neuronal cells, most of which are likely to be primary interneurones and was found at the stage of development when synaptogenesis takes place.

Although the cytoplasmic domains of the $\alpha$ - and $\beta$-neurexins are identical, the two have distinct functions linked to interactions with the presynaptic apparatus (O'Connor et al., 1993; Perin 1994; Hata et al, 1996; Butz et al., 1998; Biederer and Sudhof, 2000; Zhang, et al., 2001) and distinct extracellular ligands including neurexophilins ( $\alpha$-neurexin-specific, neuropeptide-like molecules) neuroligins ( $\beta$-neurexin-specific) and dystroglycan. The $\beta$-neurexins and neuroligins form a pair of heterophilic cell adhesion molecules (Nguyen and Sudhof, 1997) that may mediate synapse formation (Scheiffele et al, 2000) and promote post-synaptic specialisations (Graf et al, 

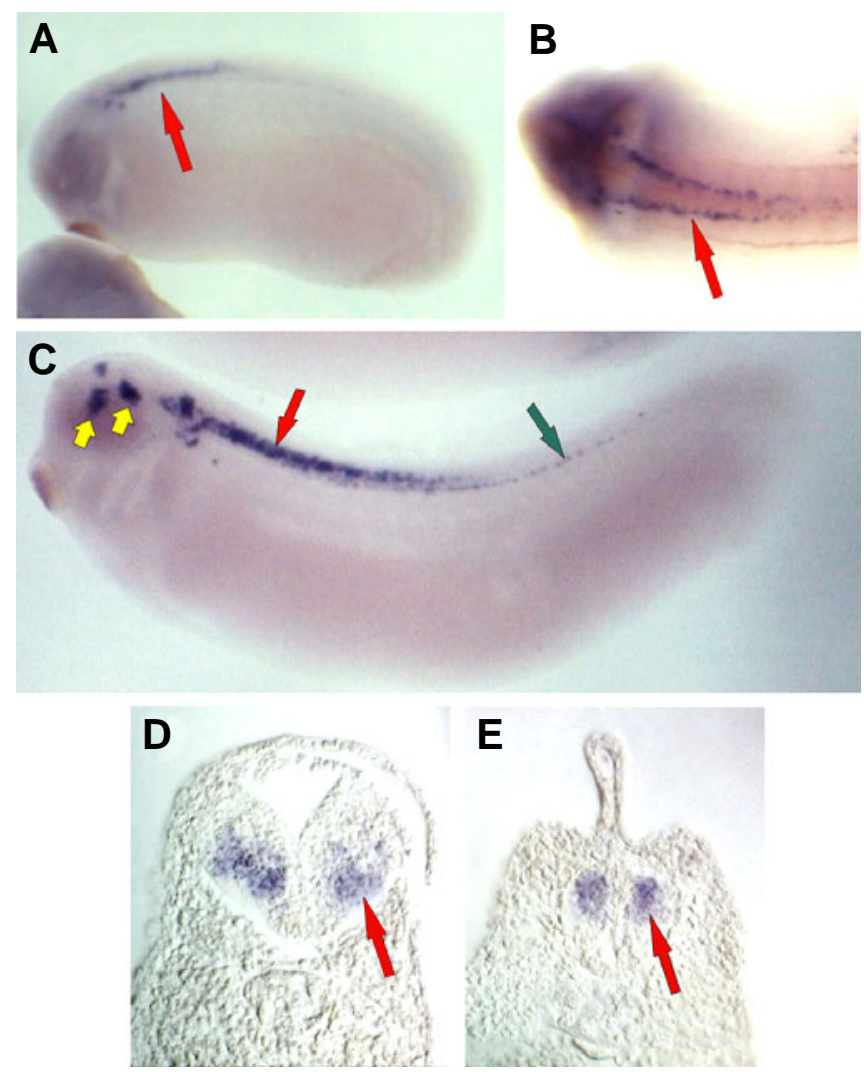

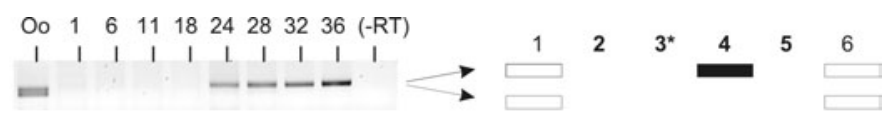

Xnrxn1 ssp1

(+)TGFQGKDCSE GLAHLMMGDQ GKEEY (-) TGFQGKDCSE -------- GKEEY

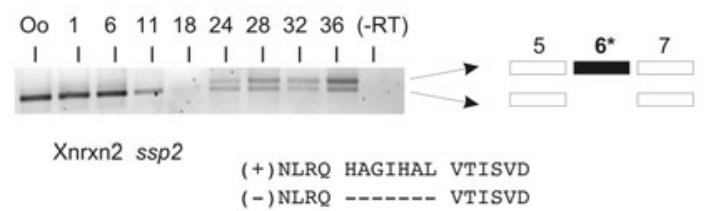

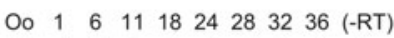

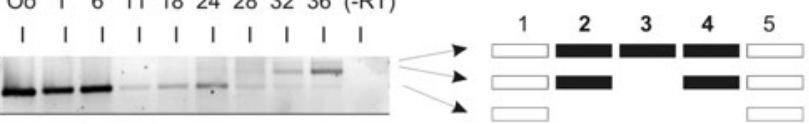

Xnrxn3 ssp 1

$(+)$ FCSE DANDIP GLAHLMIDDQ VRSK AREE

(-)FCSE DANDIP ------- VRSK AREE

(0) FCSE -

Fig. 5 (Left). Nrxn III $\alpha$ is expressed in a subset of neural cells. (A,B) In situ hybridisation with an $\alpha$-nrxn III-specific probe identifies a row of primary neurons at stage 24(A), that lies either side of the midline towards the anterior end of the embryo. (C) By the tailbud stage, expression resolves into two stripes either side of the midline (red and green arrows). Those arrowed in red are located in the position expected for primary interneurons. The second band is located more ventrally. Within the head there are discrete areas of expression (yellow arrows). (D,E) Transverse sections at the level of the hindbrain (D) and anterior spinal cord (E). nrxnllla expression (red arrows) is restricted to the lateral neural tube.

Fig. 6 (Right). The alternative splicing of the $\boldsymbol{\alpha}$-neurexins is under developmental regulation. Nrxn I was found to have isoforms based on alternative splicing at site 1 (ssp 1), which generated two forms in the oocyte but only one in the tailbud embryo. The larger form included the equivalent of mouse exon 4 but lacked the other exons at ssp1. The smaller transcript lacked all the variable exons at ssp1. Nrxn II was also found in two forms based on alternative splicing at splice site 2. The larger form included exon 6 and was detected only in the tailbud and later stages. Nrxn III existed in three isoforms varying at splice site 1. Again the forms showed a developmental profile with the longer form being detectable only in the tailbud and later stages. Left-hand side: representative images of PCR products. Right-hand side: diagrammatic representations of splice variants. Open boxes: non-variant exons present in Xenopus, filled boxes, variant exons present in Xenopus transcripts. Predicted protein sequences resulting from alternative splicing shown underneath. Numbering identifies equivalent exon in the mouse (Tabuchi and Sudhof, 2002) and bold indicates range of splice site. Exons labelled with an asterisk can occur in more than one form in the mouse.

2004). Less is known of the functions of $\alpha$-neurexins, except that they mediate the assembly of presynaptic terminals and couple $\mathrm{Ca} 2+$ channels to synaptic vesicle exocytosis (Dean et al, 2003; Missler et al, 2003), but this does not explain the need for their extensive diversity. Single and double knockouts of specific neurexin genes are viable but have distinct phenotypes and mortality rates. For example, single $\alpha$-neurexin III knockouts exhibit a lower survival rate than some double knockout mice (Missler et al, 2003) but mice lacking all three $\alpha$-neurexins die shortly after birth (Missler et al, 2003). The expression of nrxnlll $\alpha$ in a specific subset of cells in the frog is consistent with limited functional redundancy and can be examined by gene knock-down using antisense morpholino oligonucleotides.

During mouse development, the neurexins are expressed at stages associated with synaptogenesis (Puschel and Betz 1995) (Górecki et al, 1999). This is also the case in the frog and is consistent with a role for neurexins in aspects of synaptogenesis suggested by changes in $\alpha$-neurexin expression during synaptic remodelling in the adult mouse brain
(Górecki et al, 1999). Perhaps the most remarkable feature of the neurexins is the enormous variability conferred by alternative splicing. This has been taken as an indication that the neurexins are involved in differential cell-recognition within the nervous system. We have found strong conservation of alternative splice site positions and of predicted alternative sequences between mammalian and amphibian $\alpha$-neurexins, which suggests that the isoforms are of functional importance. These results are in good agreement with the observed variation in neurexin alternative splicing in mammals (Puschel and Betz, 1995) and the high conservation of splice variants between chicken and mammals (Patzke and Ernsberg, 2000) However, the alternative splicing of amphibian neurexin primary transcripts in the embryo generates much less diversity than seen in the mouse. This may relate to the limited diversity of cell types within the amphibian primary nervous system and its simpler connectivity.

Surprisingly, all three $\alpha$-nrxns are expressed in oocytes and nrxnlll $\alpha$ shows oocyte-specific splicing. This may indicate an 
additional role for neurexins outside the CNS. Calcium ions are important second messengers at fertilization (reviewed in Williams 2002), however, the known targets for calcium signalling in oocyte activation are different to those associated with neurexins at the synapse. It is not yet known whether neurexins are expressed in mammalian oocytes, but in neurexin knockout mice there is no reported abnormality in fertilization (Missler et al, 2003). This may suggest that the involvement of $\alpha$-nrxn in frog oocyte function is not conserved or that other proteins compensate for the lack of $\alpha$-neurexins in mammalian oocytes.

Xenopus provides a model system in which there is a significant understanding of the origins of neural cell types and, at a later stage, of the function of primary neurons in the circuits that control early behaviour. Whilst it remains to be seen whether the $\beta$-neurexins show specific patterns of expression in the frog, we have shown that $\alpha$-nrxns are expressed in primary neurones across the stages when individual neurones transform into a complex neuronal circuit and are subject to alternative splicing

\section{TABLE 2}

\section{SEQUENCES OF PRIMERS USED FOR PCR ANALYSES OF $\alpha$-NEUREXIN TRANSCRIPTS}

\section{A}

\begin{tabular}{|c|c|c|}
\hline Primer name & Primer sequence & Position \\
\hline Degenerate & 5'-CCGATCGATAYGCNATGTAYCAARTAY-3' & To peptide \\
\hline primer 5 ' & Clal & YAMYKY \\
\hline Degenerate & 5'-CCGCTCGAGRTARTAYTCYTTRTCYTTRTTYTT-3' & To peptide \\
\hline primer 3' & Xhol & KNKDKEYY \\
\hline X.laevis $\mathrm{N} \times 1 \alpha \mathrm{N} 1$ & 5'- GGCAATCTGGAAAATCAAC -3' & -19 to -12 \\
\hline X.laevis $\mathrm{N} \times 1 \alpha \mathrm{N} 2$ & 5'-AATAACTGGCATGATGTGAAAGT -3' & $327-334$ \\
\hline X.laevis $\mathrm{Nx} 1 \alpha \mathrm{N} 3$ & 5'-AACACCTGGCATGATGTGAAAGT-3' & $422-439$ \\
\hline$X . / a e v i s \mathrm{Nx} 1 \alpha \mathrm{C} 1$ & 5'-GCACTACTGGGTTGTTTTTCC-3' & $1442-1449$ \\
\hline X.laevis $\mathrm{Nx} 1 \alpha \mathrm{C} 2$ & 5'-CCCAGGTATCCTGTTACAATCACAGA-3' & $665-672$ \\
\hline X.laevis $\mathrm{Nx} 1 \alpha \mathrm{C} 3$ & 5'-CAACTGAACATATCCCACCATTAAGACA-3' & $210-217$ \\
\hline X.laevis $\mathrm{N} \times 3 \alpha \mathrm{N} 1$ & 5'-GCСАСССТСАСАСТСТTСТС-3' & -20 to -12 \\
\hline$X$. laevis $\mathrm{N} \times 3 \alpha \mathrm{N} 2$ & 5'-AGGCTAATGATGGAGAGTGGT-3' & $540-547$ \\
\hline$X$. laevis $\mathrm{N} \times 3 \alpha \mathrm{N} 3$ & 5'-CATCACGGGATGGATTTCAG-3' & $1014-1021$ \\
\hline X.laevis $\mathrm{N} \times 3 \alpha \mathrm{C} 1$ & 5'-GCTTGCTGTGGTTGCCCTTAGAAC-3' & $1448-1455$ \\
\hline X.laevis $\mathrm{N} \times 3 \alpha \mathrm{C} 2$ & 5'-CTCTCCGAACCACACGAACAG-3' & $782-789$ \\
\hline X.laevis $\mathrm{N} \times 3 \alpha \mathrm{C} 3$ & 5'-CTGCAGTGCTTGGGCTGCCC-3' & 388-395 \\
\hline
\end{tabular}

B

\begin{tabular}{llll} 
& Primer name & Primer sequence & Position \\
\hline X.laevis Nx1 $\alpha$ & & & \\
Ssp1 & Xnr1 ssp1 F & 5'-GTGTGCCCAAGACCAAGTGT-3' & $204-211$ \\
& Xnr1 ssp1 R & 5'-GAGACGGCTCCATTTTTCAA-3' & $297-394$ \\
Ssp2 & Xnr1 ssp2 F & 5'-CCCTTCAGAGGAATGGACTG-3' & $277-284$ \\
& Xnr1 ssp2 R & 5'-AAAAGTCATCTGATCCCAGCA-3' & $369-376$ \\
Ssp3 & Xnr1 ssp3 F & 5'-GAGCTGGATGCAGGAAGAGT-3' & $732-738$ \\
& Xnr1 ssp3 R & 5'-GCCATTTGACCTGTCATTCC-3' & $792-798$ \\
X.laevis Nx2 $\alpha$ & & & \\
Ssp1 & Xnr2 ssp1 F & 5'-TGGAAGAACGGGAGAGGTTA-3' & $202-208$ \\
& Xnr2 ssp1 R & 5'-AGCATAAGGGGATTTCGTTG-3' & $292-299$ \\
Ssp2 & Xnr2 ssp2 F & 5'-CCTGTCAATGGCAAGTTCAA-3' & $332-339$ \\
& Xnr2 ssp2 R & 5'-TGAAGCAAGACGGGAAAGTT-3' & $422-429$ \\
Ssp3 & Xnr2 ssp3 F & 5'-GCTGCGGTGTTGAGTTATGA-3' & $702-708$ \\
& Xnr2 ssp3 R & 5'-TCCACAGTGACGTTGTCCAC-3' & $811-818$ \\
X.laevis Nx3 $\boldsymbol{\alpha}$ & & & \\
Ssp1 & Xnr3 ssp1 F & 5'-ATGGAAACACAGAGCCAAGG-3' & $181-188$ \\
& Xnr3 ssp1 R & 5'-GAGTCCGTTCCTTTGTCGAG-3' & $293-310$ \\
Ssp2 & Xnr3 ssp2 F & 5'-CGACAAAGGAACGGACTCAT-3' & $293-310$ \\
& Xnr3 ssp2 R & 5'-TTTAGGCAGCCCATGAAGTT-3' & $403-410$ \\
Ssp3 & Xnr3 ssp3 F & 5'-CTGAGGCAGAGGATGTTTCC-3' & $708-715$ \\
& Xnr3 ssp3 R & 5'-CTCCGTCATGAACCCAGTCT-3' & $818-825$ \\
& &
\end{tabular}

(A) Primers used for PCR amplification of specific neurexin transcripts.

(B) Primer pairs used for analyses of alternative splicing of neurexin transcripts. that generates a range of neurexin isoforms. This is likely to add to the significance of Xenopus as a model system for the analysis of neurogenesis.

\section{Materials and Methods}

\section{Xenopus embryos}

Xenopus embryos were maintained in $0.1 \mathrm{x}$ MBS according to Gurdon (1977) and developmental stages established according to Nieuwkoop and Faber (1994). Oocytes were prepared from isolated ovaries by collagenase treatment (Guille, 1999).

\section{RNA extraction and first strand synthesis}

Adult $X$. laevis brains, or embryos at specific stages, were homogenised in NETS buffer (0.3M NaCl, $1 \mathrm{mM}$ EDTA, $20 \mathrm{mM}$ Tris $\mathrm{HCl}$ $\mathrm{pH} 7.6$ and $1 \%$ SDS). The homogenates extracted sequentially with phenol, phenol/chloroform and finally chloroform and the aqueous phase precipitated with ethanol at $-20^{\circ} \mathrm{C}$. DNA was removed with RNase-free deoxyribonuclease I (Roche), RNA recovered by phenol extraction and ethanol precipitation and resuspended in nuclease-free water. Approximately $5 \mu \mathrm{g}$ of total RNA was converted to cDNA using random hexamers and Superscript II reverse transcriptase (Invitrogen). Aliquots of cDNA were used for PCR analysis using Taq polymerase (Qiagen, Crawley, UK).

Degenerate primers were designed based on the available neurexin sequences to amplify cDNA encoding the majority of the highly conserved cytoplasmic domains of the neurexins, between the sequences YAMYKY and KNKDKEYY (amino acids: 1532 - 1537 and 1579 - 1586, respectively, amino acid numbering according to mouse $\mathrm{Nx}$ III sequence; accession No. BC060719). The primer sequences are listed in Table 2.

PCR products were resolved by electrophoresis in $2 \%(\mathrm{w} / \mathrm{v})$ agarose gels (Sigma) or $3 \%(\mathrm{w} / \mathrm{v})$ high-resolution Metaphor (Flowgen) gels and visualised by ethidium bromide staining. The PCR products were cloned (in $\mathrm{PGEM} \AA$-T Easy vector; Promega) and their identity was confirmed by DNA sequencing of both strands (commercial sequencing, Lark, UK). Control PCR experiments with samples prepared without reverse transcriptase were performed to ensure that genomic DNA contamination did not contribute to the PCR amplification.

\section{DNA library screening and characterisation of clones}

Approximately $2 \times 10^{5}$ phage plaques of a ZAPII $X$. laevis tadpolestage cDNA library (M. King, Indiana University) was screened using $X$. laevis neurexin probes generated by PCR with the degenerate primers described above. The inserts from the positive clones were subcloned into the pBluescript $\left(\mathrm{SK}_{+}\right)$vector (Stratagene) and sequenced. In addition, $X$. laevis nrxnl $\alpha$ and nrxnlll $\alpha$ clones were obtained by RTPCR amplification of RNA from frog brain based on sequences deposited in the Xenopus EST database (NCBI, BJ096899, BG486990). The primer sequences are listed in Table 2 . The resulting amplicons were cloned in the PGEM®-T Easy vector (Promega) and sequenced.

\section{Whole mount in situ hybridisation}

Anti-sense and sense digoxygenin-labelled $X$. laevisneurexin cRNA probes were transcribed in vitro and used for wholemount in situ hybridisation (Harland, 1991; Baker et al., 1995) with MEMFA-fixed Xenopus oocytes and embryos at a range of developmental stages. Samples were bleached in $10 \%$ hydrogen peroxide in 1 XPBS to remove pigment and then washed extensively in PBS before prehybridisation at $60^{\circ} \mathrm{C}$ for six hours. The embryos were then hybridised overnight with the DIG probe and subsequently washed at high stringency. Before adding the anti-DIG antibody the samples were blocked for 6 hours at room temperature. Alkaline phosphatase activity was detected using NBT and BCIP. Samples were made transparent in Murray's clear 
before photography. Some stained embryos were embedded in PEDS wax (Goldstone and Sharpe 1998) and analysed as $15 \mu \mathrm{m}$ microtome sections. Other stained embryos were embedded in $40 \%$ gelatin, fixed for five days in $20 \%$ paraformaldehyde at $4^{\circ} \mathrm{C}$ before being cut into $50 \mu \mathrm{m}$ sections on a vibratome.

\section{Acknowledgments}

This work was supported by the Wellcome Trust grant No. 066925. We thank Kerry Riley for help with the initial RT-PCR experiments.

\section{References}

ALBRIGHT, T.D., JESSELL, T.M., KANDEL, E.R., POSNER, M.I., (2000). Neural science: a century of progress and the mysteries that remain. Neuron. 25: S155.

BAKER, C.V.H, TORPEY, N.B., SHARPE, C.R., HEASMAN, J., WYLIE, C.C., (1995). A Xenopus c-kit related receptor tyrosine kinase expressed in migrating stem cells of the lateral line system. Mech. Dev. 50: 217-228.

BIEDERER, T AND SUDHOF, T.C. (2000). Mints as Adaptors: direct binding to neurexins and recruitment of Munc18. J Biol. Chem. 275: 39803-6.

BUTZ, S., OKAMOTO, M., SUDHOF, T.C. (1998). A tripartite protein complex with the potential to couple synaptic vesicle exocytosis to cell adhesion in brain. Cell. 94: 773-82.

DEAN, C., SCHOLL, F.G., CHOIH, J., DEMARIA, S., BERGER, J., ISACOFF, E., SCHEIFFELE, P., (2003). Neurexin mediates the assembly of presynaptic terminals. Nat Neurosci. 6: 708-16.

GOLDSTONE, K. AND SHARPE, C.R., (1998). The expression of XIF3 in undifferentiated anterior neurectoderm, but not in primary neurons, is induced by the neuralizing agent noggin. Int. J. Dev. Biol. 42: 757-762.

GÓRECKI, D.C., DERRY, J.M., BARNARD, E.A., (1994). Dystroglycan: brain localisation and chromosome mapping in the mouse. Hum Mol Genet. 3: 158997.

GÓRECKI, D.C., SZKLARCZYK, A., LUKASIUK, K., KACZMAREK, L., SIMONS, J.P., (1999). Differential Seizure-Induced and Developmental Changes of Neurexin Expression. Mol. Cell. Neurosci. 13: 218-227.

GRAF, E.R, ZHANG, X., JIN, S.X., LINHOFF, M.W., CRAIG, A.M. (2004). Neurexins Induce Differentiation of GABA and Glutamate Postsynaptic Specializations via Neuroligins. Cell. 119: 1013-26.

GUILLE, M. (1999). Molecular Methods in Developmental Biology (Xenopus and Zebrafish). In: Methods in Molecular Biology, 127.

GURDON, J.B. (1977). Methods for nuclear transplantation in Amphibia. Methods Cell Biol. 16: 125-139.

HARLAND, R.M. (1991). In situ hybridisation: an improved wholemount method for Xenopus embryos. Methods Cell Biol. 36: 685-95.

HATA, Y., BUTZ, S. and SUDHOF, T.C. (1996). CASK: a novel dlg/PSD95 homolog with an $\mathrm{N}$-terminal calmodulin-dependent protein kinase domain identified by interaction with neurexins. J. Neurosci. 16: 2488-2494.

JACOBSEN, M. AND HUANG, S. (1985). Neurite outgrowth traced by means of horseradish peroxidase inherited from ancestral cells in frog embryos. Dev. Biol 110: 102-111

MISSLER, M., SUDHOF, T.C. (1998). Neurexins: three genes and 1001 products. Trends Genet. 14: 20-6.

MISSLER, M., ZHANG, W., ROHLMANN, A., KATTENSTROTH, G., HAMMER, R.E., GOTTMANN, K., SUDHOF, T.C. (2003). Alpha-neurexins couple Ca2+ channels to synaptic vesicle exocytosis. Nature. 423: 939-48.

NGUYEN, T., SÜDHOF, T.C. (1997). Binding properties of neuroligin 1 and neurexin 1 beta reveal function as heterophilic cell adhesion molecules. J. Biol. Chem. 272: 26032-9.

NIEUWKOOP, P.D., FABER J. (1994). Normal table of Xenopus laevis (Daudin) 2nd Edition, North Holland Publishing Co, Amsterdam.
O'CONNOR, V.M., SHAMOTIENKO, O., GRISHIN, E., BETZ, H. (1993). On the structure of the 'synaptosecretosome'. Evidence for a neurexin/ synaptotagmin/ syntaxin/Ca2+ channel complex. FEBS Lett. 326: 255-60.

PATZKE, H., ERNSBERGER, U. (2000). Expression of neurexin lalpha splice variants in sympathetic neurons: selective changes during differentiation and in response to neurotrophins. Mol. Cell. Neurosci.15: 561-72.

PERIN, M.S. (1994). The $\mathrm{COOH}$ terminus of synaptotagmin mediates interaction with the neurexins. J Biol Chem. 269: 8576-81.

PUSCHEL, A.W., BETZ, H. (1995). Neurexins are differentially expressed in the embryonic nervous system of mice. JNeurosci. 15: 2849-56.

ROBERTS, A., CLARKE, J.D.W. (1982). The neuroanatomy of an amphibian spinal cord. Phil. Trans Roy. Soc. Lond. B 296: 195-212.

ROWEN, L., YOUNG, J., BIRDITT, B., KAUR, A., MADAN, A., PHILIPPS, D.L., QIN, S., MINX, P.,WILSON, R.K., HOOD, L., GRAVELEY, B.R. (2002). Analysis of the human neurexin genes: alternative splicing and the generation of protein diversity. Genomics. 79: 587-97.

SCHEIFFELE, P., FAN, J., CHOIH, J., FETTER, R., SERAFINI, T. (2000). Neuroligin Expressed in Non-neuronal Cells Triggers Presynaptic Development in Contacting Axons. Cell. 101: 657-669.

SCHMUCKER, D., CLEMENS, J.C., SHU, H., WORBY, C.A., XIAO, J., MUDA, M., DIXON, J.E., ZIPURSKY, S.L. (2000). Drosophila Dscam is an axon guidance receptor exhibiting extraordinary molecular diversity. Cell. 101: 671-84.

SHARPE, C.R., GOLDSTONE, K. (1997). Retinoid receptors promote primary neurogenesis in Xenopus. Development 124: 515-523.

SHARPE, C.R., GOLDSTONE, K. (2000). Retinoid signalling acts during the gastrula stages to promote primary neurogenesis. Int. J. Dev. Biol. 44: 463-470.

SHARPE, C.R., GOLDSTONE, K. (2000). The control of Xenopus embryonic primary neurogenesis is mediated by retinoid signalling in the neurectoderm. Mechanisms of Development 91 : $69-80$

SONG, J.Y., ICHTCHENKO, K., SÜDHOF, T.C., BROSE, N. (1999). Neuroligin 1 is a postsynaptic cell-adhesion molecule of excitatory synapses. Proc Nat/Acad SciUS A. 96: 1100-5.

SUGITA, S., SAITO, F., TANG, J., SATZ, J., CAMPBELL, K., SUDHOF, T.C. (2001). A stoichiometric complex of neurexins and dystroglycan in brain. J. Cell. Biol. 154: 435-45.

SUN, H.B., YOKOTA, H., CH, X.X., XU, Z.C. (2000). Differential expression of neurexin mRNA in CA1 and CA3 hippocampal neurons in response to ischemic insult. Brain Res. Mol. Brain Res. 84: 146-9.

TABUCHI, K., SUDHOF, T.C. (2002). Structure and evolution of neurexin genes: insight into the mechanism of alternative splicing. Genomics 79: 849-59.

ULLRICH, B., USHKARYOV, Y.A., SÜDHOF, T.C. (1995). Cartography of neurexins: more than 1000 isoforms generated by alternative splicing and expressed in distinct subsets of neurons. Neuron 14: 497-507.

USHKARYOV, Y.A., HATA, Y., ICHTCHENKO, K., MOOMAW, C., AFENDIS, S., SLAUGHTER, C.A., SÜDHOF, T.C. (1994). Conserved domain structure of beta-neurexins. Unusual cleaved signal sequences in receptor-like neuronal cell-surface proteins. J. Biol. Chem. 269, 11987-92.

USHKARYOV, Y.A., PETRENKO, A.G., GEPPERT, M., SÜDHOF, T.C. (1992). Neurexins: synaptic cell surface proteins related to the alpha-latrotoxin receptor and laminin. Science 257: 50-6.

WILLIAMS, C.J. (2002) Signalling mechanisms of mammalian oocyte activation. Hum Reprod. Update. 8: 313-21.

ZHANG, Y., LUAN, Z., LIU, A., HU, G. (2001). The scaffolding protein CASK mediates the interaction between rabphilin3a and beta-neurexins. FEBS Lett. 497: 99-102.

ZITO, K., SVOBODA, K. (2002). Activity-dependent synaptogenesis in the adult Mammalian cortex. Neuron 35: 1015-7.

Received: July 2005

Reviewed by Referees: September 2005

Modified by Authors and Accepted for Publication: October 2005 